\title{
ERRATUM: AN ANALYSIS OF TOTALLY CLAIRVOYANT SCHEDULING
}

Subramani, K., "An Analysis of Totally Clairvoyant Scheduling" appeared in Journal of Scheduling, vol. 8, 2, pp. 113-133 (2005).

The following misprints appeared in the print version of the paper. The publisher apologises for these errors.

(a) Page 114, Line 15 from the top-It should read "real-time systems" and not real-time systems'.

(b) Page 121, Line 11 from the bottom-It should read, "It follows that for all $\overrightarrow{\mathbf{e}} \in \mathbf{E}, \mathcal{G}_{\overrightarrow{\mathbf{e}}}$ does not have".

(c) Page 121, Line 9 from the bottom-It is Cormen, Leiserson, and Rivest, 1992.

(d) Page 125-The following table should be used.

Table 1. Computing the type of a path from the types of its sub-paths

\begin{tabular}{lll}
\hline$v_{i} \leadsto u_{k}$ & $v_{k} \leadsto v_{j}$ & $v_{i} \leadsto v_{j}$ \\
\hline Type I & Type I & Type I \\
Type I & Type II & Type IV \\
Type I & Type III & Type I \\
Type I & Type IV & Type IV \\
Type II & Type I & Type III \\
Type II & Type II & Type I (if $j=i$ ) \\
Type II & Type II & Type II (if $j \neq i$ ) \\
Type II & Type III & Type III \\
Type II & Type IV & Type I (if $j=i$ ) \\
Type II & Type IV & Type II (if $j \neq i$ ) \\
Type III & Type I & Type III \\
Type III & Type II & Type I (if $j=i$ ) \\
Type III & Type II & Type II (if $j \neq i$ ) \\
Type III & Type III & Type III \\
Type III & Type IV & Type I (if $j=i$ ) \\
Type III & Type IV & Type II (if $j \neq i$ ) \\
Type IV & Type I & Type I \\
Type IV & Type II & Type IV \\
Type IV & Type III & Type I \\
Type IV & Type IV & Type IV \\
\hline & & \\
\hline
\end{tabular}


(e) Page 127, Equation (12)-

$$
\begin{aligned}
d_{i j}^{k}(I I I)= & w_{i j}(I I I), \quad \text { if } k=0 \\
= & \min _{\mathbf{E}}\left\{d_{i j}^{k-1}(I I I), d_{i k}^{k-1}(I I)+d_{k j}^{k-1}(I), d_{i k}^{k-1}(I I)+d_{k j}^{k-1}(I I I),\right. \\
& \left.d_{i k}^{k-1}(I I I)+d_{k j}^{k-1}(I), d_{i k}^{k-1}(I I I)+d_{k j}^{k-1}(I I I)\right\}, \quad \text { otherwise }
\end{aligned}
$$

(f) Page 128, Line 9 from the bottom-Replace "relaxed Pinedo (1995)" with "relaxed (see Pinedo, 1995)." 